Compte rendu de thèse

Caroline Blondy, Les territoires touristiques

polynésiens. Une lecture géographique de la participation de la société locale au système touristique

Thèse de doctorat en géographie, université de Bordeaux III, dirigée par François Bart (soutenue le 17 novembre 2010)

\title{
Caroline Blondy
}

\section{OpenEdition}

\section{Journals}

Édition électronique

URL : http://journals.openedition.org/tourisme/521

DOI : 10.4000/tourisme.521

ISSN : 2492-7503

Éditeur

Éditions touristiques européennes

Édition imprimée

Date de publication : 1 juin 2011

Pagination : 87-90

ISSN : 2109-5671

\section{Référence électronique}

Caroline Blondy, "Caroline Blondy, Les territoires touristiques polynésiens. Une lecture géographique de la participation de la société locale au système touristique », Mondes du Tourisme [En ligne], 3 | 2011, mis en ligne le 30 septembre 2015, consulté le 10 décembre 2020. URL : http://journals.openedition.org/ tourisme/521 ; DOI : https://doi.org/10.4000/tourisme.521

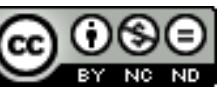

Mondes du tourisme est mis à disposition selon les termes de la licence Creative Commons Attribution - Pas d'Utilisation Commerciale - Pas de Modification 4.0 International. 


\title{
Les territoires touristiques polynésiens Une lecture géographique de la participation de la société locale au système touristique
}

\author{
Thèse de doctorat en géographie, université de Bordeaux III \\ dirigée par François Bart \\ (soutenue le 17 novembre 2010) \\ CAROLINE BLONDY \\ [caroline.blondy@univ-Ir.fr]
}

5 n s’appuyant sur la définition systémique du tourisme forgée par l'équipe Mit (2002, 2003), la thèse Les territoires touristiques polynésiens : une lecture géographique de la participation de la société locale au système touristique montre que tout d'abord que le tourisme conduit les touristes à un "déplacement spatial” (découverte de lieux différents) et à un "déplacement social" (rencontre d'individus différents : la société locale et les autres touristes) ; elle s'attache ensuite à donner une lecture géographique de l'interaction entre tourisme et société locale.

Les travaux scientifiques analysant l'interaction entre tourisme et société locale considèrent, en général, que touristes et société locale sont deux catégories distinctes et homogènes. Ils montrent, avec une approche essentiellement économique, le tourisme comme une activité impactant les sociétés locales. Notre thèse a pour premier but de nuancer cette lecture très stéréotypée.

En Polynésie française, à côté des touristes internationaux aux profils très variés - des différences notables se profilent entre un touriste japonais et un touriste métropolitain en visite dans sa famille résidente -, il existe aussi des touristes domestiques. Nous préférons le terme de touristes domestiques à celui de touristes intérieurs dans la mesure où, comme l'a déjà montré Emmanuelle Peyvel (2009), l'étymologie de cet adjectif (domus en latin) souligne le processus d'appropriation des lieux touristiques. Ici, c'est le processus d'appropriation par les touristes résidant en Polynésie française que nous nous employons à mettre en évidence.

Parallèlement, la société locale n'est pas homogène. Dans ces terres anciennement colonisées, ayant connu des mouvements migratoires, la société locale est composite, métissée, plurielle. Elle associe une communauté polynésienne, métisse ou demie, blanche ou popa'a, et chinoise. Au final, elle est composée de populations aux modes de vie différents au regard de leurs référents socioculturels (alimentation, langue, pratiques du travail, religion, etc.). Il est donc probable que les individus qui composent la société locale ont des représentations et des pratiques spatiales différentes, et qu'ils se positionnent différemment face au tourisme et aux touristes. La Polynésie française, espace pluriarchipélagique vaste comme l'Europe, est également marquée par la discontinuité territoriale, et il n'y a pas une identité polynésienne, mais des identités qui se dessinent selon les îles et les archipels. La complexité de cette société locale se lit donc également dans une pluralité multiscalaire et souligne des positionnements pluriels face au tourisme et aux touristes en fonction des îles.

Par ailleurs, les études classiques du tourisme ont une lecture de l'interaction tourisme et société locale placée sous la problématique de l'impact (Butler, 1974 ; Cazes, 1992 ; Rossel, 1984). Or cette lecture pose, elle aussi, un certain nombre de problèmes, déjà soulevés par certains sociologues, anthropologues (Michaud, 200I ; Picard, 200 I) ou géographes, comme ceux de l'équipe Mit (2002, 2003). Le tourisme est souvent mis sur le banc des accu- 
sés. Les impacts économique, social, culturel, spatial et environnemental sont souvent placés sous le signe de la destruction ou de la déstructuration. Si les revenus et les emplois créés par le tourisme ne sont pas fondamentalement remis en cause - la saisonnalité et la monoactivité étant néanmoins souvent dénoncées -, le tourisme est souvent présenté comme un phénomène subi par les populations locales, le touriste étant l'étranger venant perturber un espace. Touristes et sociétés locales sont alors étudiés dans un rapport d'opposition entre autochtones et allochtones. Le tourisme serait donc imposé et subi par les populations locales.

Notre thèse prend le contre-pied de cette problématique de l'impact déstructurant et analyse les modes de participation de la société locale au système touristique. Une lecture de la société locale polynésienne impactée par le tourisme, opposant touristes et habitants, serait une schématisation simpliste. Il n'y a pas un, mais des tourismes en Polynésie française (tourisme international et tourisme domestique), même si touristes internationaux et touristes "domestiques" utilisent des lieux souvent identiques et si leurs pratiques sont parfois proches. Il y a donc non seulement une hétérogénéité, mais aussi une perméabilité, entre les catégories "touristes" et "société locale".

Comment la société locale prendelle part au système touristique ? Quelles sont les modalités de sa participation au système touristique ? En quoi cette participation de la société locale au système touristique est-elle un révélateur des processus d'appropriation de l'espace et de disparités socio-spatiales?

La première partie de la thèse fait le point sur la place de la société locale dans la mise en tourisme des lieux, grâce à l'analyse croisée des archives territoriales, des travaux de Gilles Blanchet (|98|), des guides de voyages (de l'époque proto-touristique à nos jours), de récits autobiographiques et littéraires, des études de développement touristique, du Mémorial polynésien (Danielsson, 1977) et des entretiens avec acteurs institutionnels et professionnels. La société locale a participé, un peu malgré elle, à la construction du mythe tahitien sur lequel s'est bâtie en partie la destination touristique mythique. Elle est, en effet, partie prenante dans le mythe, à part égale avec la dimension insulaire et tropicale des lieux. Dans la phase proto-touristique, elle a été "court-circuitée" ou "mise de côté", l'initiative du développement touristique étant alors plutôt le fait d'expatriés installés depuis plus ou moins longtemps sur le territoire polynésien. Par la suite, toutefois, elle a joué un rôle dans l'extension de l'espace touristique, dans la mesure où l'hébergement chez l'habitant demeure, dans un grand nombre d'îles, la seule solution de séjour pour les touristes. L'analyse de la structuration de l'espace touristique montre que le tourisme a renforcé les phénomènes de polarisation autour de Tahiti et de centres secondaires comme Bora Bora, Moorea, etc.

La seconde partie de la thèse étudie le jeu d'acteurs du système touristique et essaie de mettre en évidence le rôle de la société locale. Cette dernière demeure un acteur secondaire dans le jeu des acteurs du système touristique polynésien, dominé en termes décisionnels essentiellement par l'État, par le pouvoir central polynésien et par les investisseurs étrangers. La société locale reste malgré tout un acteur incontournable à travers, par exemple, le principe de l'indivision. C'est en effet elle qui possède la terre. Or cette propriété de la terre, partagée parfois en un nombre impressionnant d'héritiers, est un moyen de résistance : la vente et la location de terres pour la construction d'un hôtel ou d'un aménagement touristique peuvent tourner au vaudeville.

L'étude de la participation de la société locale au système touristique à travers l'exemple polynésien permet de dédramatiser la relation entre tourisme, touristes et sociétés locale. Il n'y a pas un rapport de force entre les touristes internationaux et les investisseurs étrangers, d'un côté, et les pauvres populations locales subissant l'assaut des premiers, de l'autre. La société locale abrite une élite économique qui participe activement aux montages financiers hôteliers. De manière plus générale, la Polynésie française, rattachée à l'une des grandes puissances mondiales, fait figure de voisin riche parmi les petits pays insulaires de la zone du Pacifique Sud, malgré des contrastes sociaux très marqués.

Enfin, dans la troisième partie de la thèse, la lecture géographique de la participation de la société locale au système touristique polynésien permet de souligner des stratégies pragmatiques et opportunistes d'au moins une partie d'entre elle. Cette lecture permet de montrer que la société locale "se montre à voir" dans cette participation, comme par un jeu de miroir. Elle révèle toute la complexité de cette société locale, à la fois dans sa dimension interne, opposant des groupes sociaux aux modes de vie et aux référents culturels très différents, et dans sa dimension spatiale, les identités insulaires se déclinant à plusieurs échelles (fenua, 
archipel, île, village, famille). Cette différenciation socio-spatiale de la société locale se révèle dans cette lecture de sa participation au système touristique ; elle explique également les différentes postures et les réponses de ces sociétés locales face au tourisme et aux touristes.

La première forme de participation des habitants au système touristique est professionnelle; elle soulève un certain nombre de paradoxes. S'il est acquis qu'il existe un décalage entre la vision positive du tourisme, considérée comme une solution d'avenir pour la Polynésie française, et la difficile projection de la population dans un métier du tourisme, ce décalage concerne essentiellement le salariat dans l'hôtellerie (qui constitue l'un des principaux créateurs d'emplois touristiques), ces derniers étant mal considérés, perçus comme contraignants et mal reconnus. Nous avons montré à travers l'exemple polynésien que la société locale, même si elle ne joue qu'un rôle secondaire dans l'organisation du système touristique polynésien, peut être un acteur "entrepreneurial", et non plus seulement un acteur salarié qui subit plus qu'il n'entreprend. Cette participation professionnelle révèle des réactions différentes entre des classes modestes, qui n'ont pas d'autres choix que de travailler dans un hôtel, et une élite économique et sociale dotée d'un "capital d'entreprenariat touristique" (Blondy, 20।0) qui, volontairement, s'implique dans le secteur touristique. L'analyse de la participation professionnelle s'est appuyée sur des entretiens auprès de directeurs d'hôtel, de personnes travaillant dans des hôtels, de prestataires d'hébergement chez l'habitant, de prestataires d'activités touristiques et de responsables d'agences de voyages.
La mise en évidence de la participation des habitants au système - en tant qu'utilisateurs des lieux touristiques - a permis de mettre l'accent sur un autre aspect, allant à l'encontre de la lecture de l'impact. Le tourisme domestique en Polynésie française et les pratiques de loisirs dans les lieux touristiques alimentent le système touristique polynésien et montrent une appropriation de ces lieux, devenus des "lieux hybrides" (Bernardie-Tahir, 2007). Trois exemples (les plages, les hôtels, les hébergements chez l'habitant) sont analysés. L'appropriation passe par une imitation, une hybridation des pratiques développées par les touristes internationaux, ou par une création de pratiques propres à la société locale. L'analyse de ces deux modalités de participation au système touristique, récréative et touristique, révèle à nouveau le même jeu de miroir ; il s'agit toujours d'une élite économique et sociale qui développe ce type de pratique. En outre, elle permet de souligner la différenciation de l'espace polynésien : l'opposition entre une Polynésie "urbaine" et une Polynésie "rurale", entre une Polynésie des centres et une Polynésie des marges, où les pratiques touristiques et récréatives de la population sont inégalement présentes. Ces disparités socio-spatiales ont été mises en évidence grâce à des entretiens menés auprès de la population pour déterminer leurs pratiques touristiques et leur position sur le tourisme, et auprès de résidents usagers de la plage et des hôtels.

Parallèlement, une immersion dans un hôtel à Bora Bora et dans une pension de famille de Tikehau a permis de mettre en place une observation participante pour analyser ces trois formes de participation (professionnelle, touristique et récréatives) au système touristique. Contre un compte-rendu des observations et une aide ponctuelle, nous avons pu obtenir une libre circulation dans la structure, une liberté d'échanges avec les différents acteurs et un hébergement gratuit qui nous ont permis d'appréhender de l'intérieur les modalités de la coprésence entre touristes et société locale.

L'étude de la participation de la société locale a permis de mettre en évidence à la fois la complexité et la dimension composite de la notion de société locale en Polynésie française. Tous les groupes sociaux la composant ne participent pas, ou participent différemment, au système touristique polynésien. Elle souligne enfin les inégalités sociales qui marquent cette société locale. La participation au système touristique polynésien (voire international) peut se révéler ainsi être un marqueur identitaire. "Dis-moi si tu participes au tourisme et je te dirais qui tu es."

Cette thèse a également permis de montrer que la coprésence entre touristes et société locale peut se décliner sous plusieurs formes. En choisissant le prisme de la nature de l'action participative de la société locale au système touristique, ce travail forge une typologie de la coprésence : la coprésence passive quand la société locale se cantonne à son rôle de société d'accueil indifférente (pas de participation active au système touristique polynésien); la coprésence professionnelle quand la société locale participe professionnellement au tourisme; la coprésence récréative quand la société locale utilise les lieux touristiques dans son territoire du quotidien pour la pratique de ses loisirs ; la coprésence circonstanciée quand elle est amenée pour de multiples raisons à utiliser des lieux 
touristiques, notamment les lieux liés à l'hébergement et aux transports ou au transit (aéroports, gares maritimes, avion, bateaux, etc.) sans y exercer des pratiques touristiques ou récréatives. Cette coprésence débouche sur des interactions d'intensité variable, allant de la rencontre de l'Autre au repli sur soi. Cette analyse des formes de coprésence nous a conduit à revisiter la notion de retranchement touristique dont les formes spatiales, l'enclavement touristique et l'île-hôtel, pourraient a priori être posées comme une tentative d'évitement ou d'atténuation de la coprésence.

En définitive, l'exemple polynésien pousse à insister sur la dissociation entre un retranchement géographique et un retranchement social. Le repli dans un lieu fermé ne conduit pas nécessairement au repli social, et n'évite pas certaines formes de coprésence et d'échanges interpersonnels. À l'inverse, un lieu ouvert n'empêche pas le repli sur soi.

Ces hôtels à la silhouette aquatique et aux bungalows sur pilotis, copiés partout dans le monde insulaire tropical, véritables chantres de la mondialisation, érigés en envahisseurs des paysages, en "voleurs d'espace", en pollueurs... ne sont pas des ghettos à touristes internationaux. Lieux de coprésence récréative et touristique, voire de coprésence circonstanciée entre les touristes internationaux et la société locale, ils font partie d'un processus de territorialisation de certains membres de la société locale et deviennent des points d'ancrage dans les processus d'appropriation de l'espace. Si les hôtels impactent le territoire polynésien, la société locale les utilise de manière très pragmatique et opportuniste - sans

RÉEÉRENCES BIBLIOGRAPHIQUES

Nathalie BERNARDIE-TAHIR, "Quand les touristes rencontrent les habitants : territoires et lieuX communs dans la ville de Zanzibar", dans Philippe Duhamel et Rémy KNAFou (dir.), Mondes urbains du tourisme, Belin, 2007.

Gilles BLANCHET, Les petites et moyennes entreprises polynésiennes : le cas de la petite hôtellerie, Travaux et documents, 136, Orstom, 1981.

Caroline BLONDY, Les territoires touristiques polynésiens : une lecture géographique de la participation de la société locale au système touristique, thèse de géographie, Bordeaux III, 2010. Richard W. BUTLER, "The social implications of tourist developments", Annals of Tourism Research, 2, 1974.

Georges CAZES, Fondements pour une géographie du tourisme et des loisirs, Amphi Géographie, Bréal, 1992.

Giorgia CERIANI, Quand la mobilité change le rapport au monde. Marocains en Méditerranée, thèse de géographie, Paris I, 2007.

Bengt et Marie-Thérèse Danielsson, Le Mémorial polynésien, IV, V, VI, Hibiscus édition, 1977.

ÉQUIPE MIT, Tourisme I. Lieux communs, Mappemonde, Belin, 2002.

ÉQUIPE MIT, Le Tourisme. Acteurs, lieux et enjeux, Belin, 2003.

Jean MiCHAUD, "Anthropologie, tourisme et sociétés locales au fil des textes", Anthropologie et sociétés, 25-2, 200।.

Michel PICARD, "Bali : vingt ans de recherches", Anthropologie et sociétés, 25-2, 200 I.

Emmanuelle PeYvel, Le tourisme domestique au Vietnam, thèse de géographie, Nice, 2009.

Pierre RosSEL, Tourisme et Tiers-Monde : un mariage blanc, Cetim, Pierre-Marcel Fabre, 1984.

qu'il y ait de jugement de valeur dans ce propos. Ce n'est pas sans ironie que l'on pourrait conclure en disant que ces hôtels sont finalement les révélateurs de la société locale et que leur fréquentation en est un marqueur identitaire.

Cette analyse de la coprésence pose également la question de la rencontre de l'Autre. Celle-ci est différente selon le projet touristique des touristes. Si certains ont besoin d'éprouver un différentiel d'altérité fort, d'autres préfèrent rester dans un "entre soi" rassurant. Ces rencontres peuvent faire l'objet d'une typologie mettant en jeu les critères des motivations, du cadre et de la nature de l'échange. La typologie souligne généralement ainsi le lien entre rencontre de l'Autre et "capital mobilitaire" (Ceriani, 2007 ; Peyvel, 2009) des touristes. Plus ce dernier est développé, plus la rencontre est facilitée.

L'exemple polynésien a pu montrer que les relations entre tourisme et sociétés locales devaient être démystifiées. Cette participation de la société locale au système touristique à travers ses pratiques restent néanmoins faible au regard de l'image touristique du lieu. Les pratiques touristiques domestiques et l'entreprenariat touristique sont à la fois freinés en raison des liens socioculturels forts avec la métropole, de la dépendance économique de cet espace ultramarin et de la manne qui en découle. Ces logiques sont, en effet, spécifiques aux espaces ultramarins français ou étrangers.

En définitive, au-delà de l'image classique de l'accueil de la vahine à l'aéroport de Faa'a avec un collier de fleurs, la rencontre de l'Autre se pose dans des termes beaucoup plus ambivalents, oscillant entre obligation, transaction, instrumentalisation, désir, plaisir et spontanéité. 